\title{
Adapting to the impact of COVID-19 on mental health: an international perspective
}

\author{
Rejish K. Thomas, MD, MSc; Raheem Suleman, MD, MSc; Marnie Mackay, PhD; \\ Lovneet Hayer, MD; Mohit Singh, MD; Christoph U. Correll, MD; Serdar Dursun, MD, PhD
}

The coronavirus disease 2019 (COVID-19) pandemic has affected the mental health of the entire population and poses unique challenges to the delivery of psychiatric services. To best respond, we must learn from evidence from past pandemics and what has been learned internationally. There is a lack of guidance on how psychiatric services should adapt to COVID-19, which has led to inconsistent reactions. This is potentially dangerous, as over- or undercompensation has a direct impact on patient and public health outcomes. In this editorial, we discuss key considerations for adapting psychiatric care to the unprecedented challenges posed by the COVID-19 pandemic. One of the most substantial effects of this pandemic has been on global mental health and addictions. Further resource allocation toward mental health will have to be addressed by policy-makers, and it is our hope this can also serve as a learning experience that could help mitigate the impact of future crises.

COVID-19, caused by infection with severe acute respiratory syndrome coronavirus 2 (SARS-CoV-2), has resulted in an unprecedented and devastating impact on patients, health care professionals, families, friends and our way of life. The impact has been even greater on those with severe mental health disorders. Psychiatric disorders share a stressdiathesis etiology, and the combination of the stress of the COVID-19 pandemic, the conditions it has forced patients into and the threat of losing biological and psychosocial treatments that kept them well could trigger psychiatric worsening and large numbers of relapses in the upcoming weeks to months. Relapse could result in significant functional decompensation and even death. Therefore, mental health professionals must learn quickly from global knowledge and devise pragmatic solutions to improve outcomes and mitigate against the broader psychiatric risk posed by COVID-19.

\section{Epidemiological estimates and psychological impact of COVID-19}

By the end of January 2020, the World Health Organization (WHO) had declared COVID-19 a public health emergency. As of May 16, 2020, the number of cases and deaths from
COVID-19 were 4649079 and 309 047, respectively, with an estimated mean infection rate of 2.5. ${ }^{1-4}$ Epidemiological data also captured the co-occurring psychological pandemic of COVID-19. Posts from more than 17000 Chinese users of Weibo (a social media platform) highlighted a significant shift toward concerns over individual and familial health and away from routine socialization and day-to-day events of normal life..$^{5}$ A survey of the Chinese public similarly showed that $53.8 \%$ of respondents rated the outbreak's psychological impact as moderate or severe, with $16.5 \%$ reporting at least moderate depressive symptoms. ${ }^{6}$ While the direct biological psychiatric manifestations of COVID-19 are yet to be determined, there is evidence that the virus may be able to cross the blood-brain barrier. ${ }^{7}$ The evidence for neuroinvasive pathology and neuroinflammation may change the future epidemiology of psychiatric disorders, an unsettling possibility that requires further research and attention.

\section{Protecting the mental health of health care workers}

Experiences from prior pandemics and from the early outbreak in China suggest that COVID-19 will also have profound mental health implications for front-line health care workers. During the severe acute respiratory syndrome (SARS) pandemic of 2003, the emotional toll resulting from direct care of infected patients, the social isolation of quarantine, and the fear of being a potential source of community spread exerted immediate and lasting mental health effects on health care workers. ${ }^{8,9}$ The average distress level among emergency department staff was 6.19 on a 10-point scale, and even 3 years later, $22.8 \%$ of hospital employees in Beijing had moderate to severe depressive symptoms. ${ }^{10}$ Twenty percent of all individuals who contracted SARS were health care workers. ${ }^{9}$ Faced with daily exposure to a changing, high-stress environment amidst the current pandemic, a considerable proportion of Chinese front-line staff has also reported symptoms of depression, anxiety, insomnia and distress. ${ }^{11}$ 
Aside from the obvious humanitarian debt owed to those who provide direct patient care during this pandemic, adequately addressing their distress has important implications with regard to reducing the risk of burnout and medical error. Provision of adequate support on a system-wide level can help greatly with stress reduction and decrease the need for more intensive interventions in the future. In China, strategies have included the adequate provision of personal protective equipment, establishment of a strict shift schedule to allow time for self-care, furnishing separate housing for those concerned about infecting family members, and ready telephone or virtual access to counsellors and other debriefing opportunities. ${ }^{12,13}$ Individuals should also be taught methods to decrease the risk of burnout and to monitor themselves for symptoms. Given that in the midst of a pandemic there can be a natural inclination to view others as a source of potential infection rather than support, one method of counteracting burnout may be the proactive use of a "buddy check-in" system, ideally delivered using video chat. ${ }^{14}$ The Chinese experience shows that even during times of crisis, front-line health care workers will avail themselves of psychological resources that are offered to them, especially when those resources are delivered in a flexible manner that is respectful of the many demands placed on medical staff. ${ }^{11}$

\section{Essential biological treatments to preserve during COVID-19}

Psychiatry has essential treatments that, if withheld, could lead to deaths or relapse requiring acute hospitalization. During a time when resources are limited and patients face acute risk of death when ill, these treatments should be protected as long as possible, comparable to other treatments such as chemotherapy and renal dialysis. Electroconvulsive therapy (ECT) is a life-saving procedure for many patients, and those requiring maintenance ECT are at risk for deterioration and suicide if not treated regularly. One of the most powerful treatments in psychiatry for depression, ECT has well-established evidence for efficacy, with an effect size of 0.91 , and can reduce the risk of suicide and future relapse. ${ }^{15-17}$ Further, intravenous ketamine has antidepressant and antisuicidal efficacy similar to ECT in treatment-resistant depression. ${ }^{18-20}$ Unfortunately, it also comes with a high relapse risk if treatments are interrupted, and few other treatments can mitigate this danger. ${ }^{21}$ The risk of deterioration for patients if these treatments are withdrawn or withheld owing to problems with access to outpatient care is compounded by the fact that other effective psychosocial treatments are made more difficult by social distancing regulations.

\section{Considerations regarding clozapine during COVID-19}

A similar problem of access to crucial care in particularly vulnerable individuals exists for often otherwise treatmentresistant patients on clozapine who can get prescriptions only after a blood draw has confirmed sufficient absolute neutrophil count (ANC). A recently issued international consensus statement outlines that during times when patients may not be able to leave the house or access phlebotomy, the frequency of ANC monitoring can be reduced from monthly to trimonthly, with dispensation of up to a 90-day supply (if it can be safely stored) for patients fulfilling all of the following criteria: continuous clozapine treatment for more than 1 year, lifetime absence of an ANC $<2000 / \mathrm{mL}$ (or $<1500 / \mathrm{mL}$ if there is history of benign ethnic neutropenia), and no safe/practical access to ANC testing. ${ }^{22}$ The experts further suggested that decisions about ANC monitoring for patients on continuous clozapine treatment for 6-12 months may be made on a case-bycase basis. The US Food and Drug Administration (FDA) has also recently issued a statement allowing for the relaxation of risk evaluation and mitigation strategies (REMS) regarding medication administration. ${ }^{23}$ Specifically, the FDA states that undergoing laboratory testing to obtain a medication subject to a REMS can put patients and others at risk for transmission of SARS-CoV-2. Therefore, they suggest that health care providers prescribing and/or dispensing such medications should consider whether there are compelling reasons not to complete these tests during the pandemic, use their best medical judgment in weighing the benefits and risks of continuing treatment in the absence of laboratory testing and imaging studies, and communicate these judgments and the risks associated with them to their patients.

\section{Telepsychiatry and COVID-19}

The COVID-19 pandemic has forced clinicians to investigate creative ways to stay home and serve their patients. To maintain outpatient care, there has been a push for more widely adopting telepsychiatry. Telepsychiatry has a long history of positive research and is well established as having equal reliability as face-to-face interactions while boasting improved outcomes owing to improved access without sacrificing the ability to form a therapeutic alliance. $^{24-26}$ Telemedicine could be a tool to overcome the large treatment gap in psychiatry, where often more than half of ill individuals are not reached by the health care system. ${ }^{27}$ The possibility of improved access was previously thwarted by roadblocks, such as stringent standards toward privacy, consent and data protection, which neglected to account for recent improvements in the safety and security of electronic communication modalities. Early reports from China suggesting that patients in isolation have actively pursued online support for mental health establish the demand. ${ }^{9,28}$

Telepsychiatry lowers the chance of virus transmission and has the potential to decrease no-show rates and reach more people who otherwise may have problems with transportation or keeping appointments. There may be hope that telepsychiatry will become more widely adopted after the COVID-19 pandemic has ceased. However, if the broader acceptance and reimbursement of telemedicine and videobased psychotherapy will extend beyond the pandemic, 
further resources should be in place to protect from potential harms. Privacy, consent and data protection should be upgraded to keep up with growing security demands. Furthermore, face-to-face visits may decline because of perceived inconvenience by the patient and relative cost. Faceto-face interactions are important in many treatment plans. A few advantages over telepsychiatry that should not be understated are the therapeutic value of leaving the house and the spontaneous therapeutic interactions that result from coming to a clinic. Also, individuals with severe mental illness who do not have a computer, smartphone or Internet access may remain disadvantaged and may have to rely on suboptimal voice-only telephone visits. In the future, efforts to reach a balance should be made, such as a mandatory in-person assessment to introduce the patient to the clinic and the clinic staff, followed by the option to continue remotely or in person.

\section{Psychological treatments for patients during COVID-19}

For individuals with and without pre-existing psychiatric illness, psychosocial interventions will also be critical to promote and maintain mental health. Cognitive behavioural therapy (CBT) is one modality that may be of use, as it can help challenge distorted thought patterns, such as catastrophizing and over-generalizing, and counteract the sense of helplessness that individuals may face. ${ }^{29}$ Moreover, CBT can be delivered via telephone, text message and guided self-help modules and remain respectful of the physical proximity restrictions that have been enacted in many jurisdictions. ${ }^{30,31}$ Evidence exists in agoraphobia, panic disorder, obsessive-compulsive disorder and posttraumatic stress disorder that short-term efficacy is similar for telecommunications and face-to-face CBT, though the longer-term efficacy requires further research. ${ }^{32-36}$ Even if not delivered in a highly formalized, structured approach, communication of CBT principles to members of the public can also be of benefit. This includes the encouragement to practise mindfulness and relaxation techniques, to focus on matters that are under one's direct control, and to keep abreast of, but not become immersed in, developments in the news. ${ }^{29}$ As previous analyses have shown that quarantine measures can exacerbate symptoms of mental illness, great care should also be taken to encourage individuals to maintain social connectedness, even in the face of physical distancing. ${ }^{37}$ In Alberta, the "Text4Hope" program provides free, CBT-derived supportive text messages to individuals who enroll simply by messaging a number, and this program could be replicated in other jurisdictions. ${ }^{38}$ If needed, patients should also be able to self-refer for more intensive psychosocial interventions, with system access coordinated through a central repository; this is the case in Britain with the "Improving Access to Psychological Therapies" program run by the National Health Service. ${ }^{39}$ These interventions may reduce the mental health impact of the pandemic, but will not supplant the need for effective pharmacotherapy.

\section{Management of substance use disorder during COVID-19}

The COVID-19 pandemic presents unique challenges for the management of people who use alcohol or drugs, and extraordinary measures are needed to support this population, which has a high baseline risk for morbidity and death. Adherence to isolation requirements is challenging for people with substance use disorder owing to social marginalization, homelessness, and the experience of substance craving and withdrawal (unpublished data, Edmonton ARCH; 2020). In acute care settings, where harm reduction approaches are not available, people with substance use disorder and influenzalike symptoms are at high risk for leaving against medical advice rather than isolating within a hospital room or receiving treatment. ${ }^{40}$ In the community, access to high-intensity programs, such as injectable opioid agonist treatment, can be life-saving. ${ }^{41}$ However, the requirements of presenting to a clinic up to 3 times a day significantly increases the risk for exposure to and spread of SARS-CoV-2. For individuals seeking residential treatment for substance use disorders, wait times may be even longer owing to some facilities practising social distancing, reducing the number of participants and declining new admissions.

Complete adoption of harm-reduction principles is an essential component of the pandemic response with respect to addictions. Consequently, on Mar. 19, 2020, Health Canada issued temporary exemptions for prescriptions of controlled substances under section 56 of the Controlled Drugs and Substances Act. ${ }^{42}$ Pharmacists are now able to extend prescriptions, transfer prescriptions to other pharmacists, permit prescribers to issue verbal orders to extend or refill prescriptions, and permit pharmacy employees to deliver controlled substances to patients. As a prescriber, it is important to consider providing prescriptions that last at least 23 days to support ongoing quarantine and social distancing measures. ${ }^{43}$ Special consideration should also be given to how the COVID-19 pandemic response could limit access to life-saving harm-reduction interventions, such as safe consumption services, and distribution of safe drug use equipment and overdose-response kits. ${ }^{43}$ Fortunately, these interventions continue to be available in many jurisdictions with the combination of support from community clinics and outreach teams. Ongoing discussion is important to ensure that these supports are available for patients who are required to self-isolate.

\section{Crisis psychiatry}

During a crisis, it takes a unique skill set to prioritize resources and creatively employ methods to sustain quality care. For example, during the Ebola crisis, there was evidence that the decrease in care to individuals with pre-existing severe disorders was harmful. ${ }^{44}$ Designated "crisis psychiatrists" could be trained for each centre to respond to the COVID-19 crisis - and any future crises - to implement timely and evidence-based interventions to improve outcomes and mitigate harm. 


\section{Conclusion}

This is no time to revive stigma. Severe mental illness is akin to other chronic medical conditions, and the absence of routine care can lead to loss of lives and a second wave of emergencies in the near future. The power of telepsychiatry, psychosocial treatments delivered via technology, adaptation of regulations to preserve continuity of care for necessary treatments and defending treatments that are necessary for vulnerable populations can help mitigate this danger. Clinicians and patients can persevere through disastrous times by keeping a distance and remaining connected.

Affiliations: From the Nuns Community Hospital, Department of Psychiatry, Edmonton, Alta., Canada (Thomas, Dursun); the Department of Psychiatry, University of Alberta, Edmonton, Alta., Canada (Thomas, Dursun, Suleman, Mackay, Hayer, Singh); the Department of Telepsychiatry, Alberta Health Services, Edmonton, Alta., Canada (Thomas, Hayer, Singh); the Neurochemical Research Unit and Bebensee Schizophrenia Research Unit, Edmonton, Alta., Canada (Mackay); the Recognition and Prevention Program, Zucker Hillside Hospital, Department of Psychiatry, Northwell Health, Glen Oaks, NY, USA (Correll); the Department of Psychiatry and Molecular Medicine, Barbara Zucker School of Medicine at Hofstra/Northwell, Hempstead, NY, USA (Correll); and the Department of Child and Adolescent Psychiatry, Charité Universitätsmedizin Berlin, Berlin, Germany (Correll).

Competing interests: R. Thomas participates on advisory boards for Sunovion and Janssen and has received speaker fees from Sunovion, Janssen, Lundbeck and Otsuka. C. Correll has been a consultant and/ or advisor to or has received honoraria from Acadia, Alkermes, Allergan, Angelini, Axsome, Gedeon Richter, Gerson Lehrman Group, IntraCellular Therapies, Janssen/J\&J, LB Pharma, Lundbeck, MedAvante-ProPhase, Medscape, Neurocrine, Noven, Otsuka, Pfizer, Recordati, Rovi, Sumitomo Dainippon, Sunovion, Supernus, Takeda, and Teva. He has provided expert testimony for Janssen and Otsuka. He served on data safety monitoring boards for Lundbeck, Rovi, Supernus and Teva. He received grant support from the Berlin Institute of health (BIH), Janssen, the National Institute of Mental Health (NIMH), Patient Centered Outcomes Research Institute (PCORI), Takeda and the Thrasher Foundation. He has received royalties from UpToDate and is also a stock option holder of LB Pharma. S. Dursun has previously acted on scientific advisory boards for Janssen Pharmaceuticals. No other competing interests were declared.

\section{References}

1. Fagiolini A, Cuomo A, Frank E. COVID-19 diary from a psychiatry department in Italy. J Clin Psychiatry 2020;81:20com13357.

2. Wu Z, McGoogan JM. Characteristics of and important lessons from the coronavirus disease 2019 (COVID-19) outbreak in China: summary of a report of 72314 cases from the Chinese Center for Disease Control and Prevention. JAMA 2020 323:1239-42.

3. Elflein J. Coronavirus (COVID-19) disease pandemic - statistics \& facts. Statista; 2020. Available: https://www.statista.com/topics/5994/ the-coronavirus-disease-covid-19-outbreak / (accessed 2020 May 16)

4. Johns Hopkins University. Johns Hopkins Coronavirus Resource Center. JHU; 2020. Available: https://coronavirus.jhu.edu/map. html (accessed 2020 Apr. 12).

5. Li S, Wang Y, Xue J, et al. The impact of COVID-19 epidemic declaration on psychological consequences: a study on active Weibo users. Int J Environ Res Public Health 2020;17:2032.

6. Wang C, Pan R, Wan X, et al. Immediate psychological responses and associated factors during the initial stage of the 2019 coronavirus disease (COVID-19) epidemic among the general population in China. Int J Environ Res Public Health 2020;17:1729.

7. Moriguchi T, Harii N, Goto J, et al. A first case of meningitis/ encephalitis associated with SARS-coronavirus-2. Int J Infect Dis 2020;94:55-8.
8. Wong TW, Yau JKY, Chan CLW, et al. The psychological impact of severe acute respiratory syndrome outbreak on healthcare workers in emergency departments and how they cope. Eur J Emerg Med 2005;12:13-8

9. Liu S, Yang L, Zhang C, et al. Online mental health services in China during the COVID-19 outbreak. Lancet Psychiatry 2020;7:e17-8.

10. Liu X, Kakade M, Fuller CJ, et al. Depression after exposure to stressful events: lessons learned from the severe acute respiratory syndrome epidemic. Compr Psychiatry 2012;53:15-23.

11. Lai J, Ma S, Wang Y, et al. Factors associated with mental health outcomes among health care workers exposed to coronavirus disease. JAMA Netw Open 2020;3:e203976.

12. Chen $\mathrm{Q}$, Liang $\mathrm{M}, \mathrm{Li} \mathrm{Y}$, et al. Mental health care for medical staff in China during the COVID-19 outbreak. Lancet Psychiatry 2020;7:e15-6.

13. Kang L, Li Y, Hu S, et al. The mental health of medical workers in Wuhan, China dealing with the 2019 novel coronavirus. Lancet Psychiatry 2020;7:e14.

14. Canadian Medical Association. Maintaining your and your family's well-being during a pandemic. CMA; 2020. Available: https://www. cma.ca/maintaining-your-and-your-familys-well-being-during -pandemic?utm_source $=$ member-comm-020420\&utm_medium =email\&utm_campaign=covid19\&utm_content=family-wellbeing -en (accessed 2020 Apr. 15).

15. Liang CS, Chung CH, Ho PS, et al. Superior anti-suicidal effects of electroconvulsive therapy in unipolar disorder and bipolar depression. Bipolar Disord 2018;20:539-46.

16. Jelovac A, Kolshus E, McLoughlin DM. Relapse following successful electroconvulsive therapy for major depression: a meta-analysis. Neuropsychopharmacology 2013;38:2467-74.

17. Geddes J, Carney S, Cowen P, et al. Efficacy and safety of electroconvulsive therapy in depressive disorders: a systematic review and meta-analysis. Lancet 2003;361:799-808.

18. Coyle CM, Laws KR. The use of ketamine as an antidepressant: a systematic review and meta-analysis. Hum Psychopharmacol 2015;30:152-63.

19. Soleimani L, Welch A, Murrough JW. Does Ketamine have rapid anti-suicidal ideation effects? Curr Treat Options Psychiatry 2015;2: 383-93.

20. Ballard ED, Ionescu DF, Vande Voort JL, et al. Improvement in suicidal ideation after ketamine infusion: relationship to reductions in depression and anxiety. J Psychiatr Res 2014;58:161-6.

21. Papakostas GI. Maintaining rapid antidepressant effects following ketamine infusion: a major unmet need. J Clin Psychiatry 2020;81: $19 r 12859$.

22. Siskind D, Honer WG, Clark S, et al. Consensus statement on the use of clozapine during the COVID-19 pandemic. J Psychiatry Neurosci 2020:45:2.

23. U.S. Department of Health and Human Services, Food and Drug Administration, Center for Drug Evaluation and Research (CDER), Center for Biologics Evaluation and Research (CBER). Policy for Certain REMS Requirements During the COVID-19 Public Health Emergency Guidance for Industry and Health Care Professionals Preface Public Comment. Rockville (MD): USFDA; 2020. Available: https://www.fda.gov/regulatory-information/search-fda -guidance-documents / policy-certain-rems-requirements-during -covid-19-public-health-emergency-guidance-industry-and (accessed 2020 May 16).

24. Hyler SE, Gangure DP, Batchelder ST. Can telepsychiatry replace in-person psychiatric assessments? A review and meta-analysis of comparison studies. CNS Spectr 2005;10:403-13.

25. Godleski L, Darkins A, Peters J. Outcomes of 98,609 U.S. Department of Veterans Affairs patients enrolled in telemental health services, 2006-2010. Psychiatr Serv 2012;63:383-5.

26. Miller EA. Telemedicine and doctor-patient communication: an analytical survey of the literature. J Telemed Telecare 2001;7:1-17.

27. Kohn R, Saxena S, Levav I, et al. The treatment gap in mental health care. Bull World Health Organ 2004;82:858-66.

28. Zhou X, Snoswell CL, Harding LE, et al. The role of telehealth in reducing the mental health burden from COVID-19. Telemed J E Health 2020;26:377-9.

29. Goldberg JF. Psychiatry's niche role in the COVID-19 pandemic. J Clin Psychiatry 2020;81: 20com13363.

30. Agyapong VIO, Juhás M, Ohinmaa A, et al. Randomized controlled pilot trial of supportive text messages for patients with depression. BMC Psychiatry 2017;17:286. 
31. Cuijpers P, Noma H, Karyotaki E, et al. Effectiveness and acceptability of cognitive behavior therapy delivery formats in adults with depression: a network meta-analysis. JAMA Psychiatry 2019;76:700-7.

32. Mcnamfe G, O'sullivan G, Lelliott P. Telephone-guided treatment for housebound agoraphobics with panic disorder: exposure vs. relaxation. Behav Ther 1989;20:491-7.

33. Lovell K, Cox D, Haddock G, et al. Telephone administered cognitive behaviour therapy for treatment of obsessive compulsive disorder: randomised controlled non-inferiority trial. BMJ 2006; 333:883-6.

34. Brenes GA, Miller ME, Williamson JD, et al. A randomized controlled trial of telephone-delivered cognitive-behavioral therapy for late-life anxiety disorders. Am J Geriatr Psychiatry 2012;20: 707-16.

35. Bouchard S, Paquin B, Payeur R, et al. Delivering cognitive-behavior therapy for panic disorder with agoraphobia in videoconference. Telemed J E Health 2004;10:13-25.

36. Swinson RP, Fergus KD, Cox BJ, et al. Efficacy of telephoneadministered behavioral therapy for panic disorder with agoraphobia. Behav Res Ther 1995;33:465-9.

37. Brooks SK, Webster RK, Smith LE, et al. The psychological impact of quarantine and how to reduce it: rapid review of the evidence. Lancet 2020;395:912-20.

38. Alberta Health Services. Novel coronavirus (COVID-19) Text4Hope. AHS; 2020. Available: https://www.albertahealthservices.ca/topics /Page17019.aspx (accessed 2020 May 17)
39. National Health Service England. Adult improving access to psychological therapies programme. NHS; 2020. Available: https://www. england.nhs.uk/mental-health/adults/iapt/ (accessed 2020 May 17).

40. Lail P, Fairbairn N. Patients with substance use disorders leaving against medical advice: strategies for improvement. J Addict Med 2018;12:421-3.

41. British Columbia Centre on Substance Use. Injectable opioid agonist treatment for opioid use disorder. BC Centre on Substance Use; 2017. Available: https://www.bccsu.ca/wp-content/uploads /2017/10/BC-iOAT-Guidelines-10.2017.pdf (accessed 2020 Apr. 12)

42. Health Canada. 4. Health Canada Subsection 56(1) class exemption for patients, practitioners and pharmacists prescribing and providing controlled substances in Canada during the coronavirus pandemic. Health Canada; 2020. Available: https://www.canada.ca/ en/health-canada/services/health-concerns/controlled-substances -precursor-chemicals / policy-regulations / policy-documents / section-56-1-class-exemption-patients-pharmacists-practitioners -controlled-substances-covid-19-pandemic.html (accessed 2020 Apr. 12).

43. British Columbia Centre on Substance Use. Risk mitigation in the context of dual public health emergencies. BC Centre on Substance Use; 2020. Available: https://www.bccsu.ca/wp-content/uploads /2020/04/Risk-Mitigation-in-the-Context-of-Dual-Public-Health -Emergencies-v1.5.pdf (accessed 2020 Apr. 3).

44. Arcos González P, Fernández Camporro Á, Eriksson A, et al. The epidemiological presentation pattern of Ebola virus disease outbreaks: changes from 1976 to 2019. Prehosp Disaster Med 2020;35:247-253.

\section{A tribute to Dr. Patricia Boksa}

"Oh, it won't be much work," she said.

Little did we know that following in Dr. Patricia Boksa's footsteps as vice-presidents of the Canadian College of Neuropsychopharmacology and then as associate editors and co-editors-in-chief of JPN would entail years of time and effort. We have been impressed with the time and prudency that Patricia dedicates to reading, researching and evaluating each submission to the journal. Even in the case of a rejection at the editorial level, she makes the effort to explain the limitations of the submission and why it is not suitable for publication.

Under Patricia's leadership through 2 terms as coeditor-in-chief (July 2009 to June 2020), and building on previous solid ground, JPN has grown in reputation, and the quality of papers submitted continues to increase. There is no doubt that while working with her predecessor, Dr. Simon Young, Patricia developed a clear editorial line for the journal with the following criteria in mind: the validity of the science and its pertinence to mental disorders. This philosophy has been "osmotically" transmitted by Patricia to us, reflecting her mastery of this art.

Whoever works with Patricia, from the most junior to the most senior, will immediately perceive her collegial approach, her care for the development of each one, and her excellent advice. If JPN has prospered, it is largely because of the efforts and great working relationship that Patricia developed with every member of the team, and the complementary expertise with editorial board members. It has been delightful to work with her, discussing suitability of manuscripts to the journal, appropriateness of their methodologies and their relevance to the evolution of psychiatric neuroscience.

Remarkably, although Patricia trained as a pharmacologist and dedicated her career to successfully researching basic biological mechanisms underlying serious mental disorders, in the last 5 years she engaged in clinical work dedicated to improve youth mental health, particularly that of Indigenous youth. In doing so, Patricia exemplifies the engagement of a scientist who makes a difference in her community, devoting particular attention to the most vulnerable.

While her leadership at the journal will be missed, we hope to count on her advice from time to time. Best wishes for the future, Patricia.

Ridha Joober and Paul Albert, co-editors-in-chief, JPN 